\title{
Short-Term Performance of Mitral Valve Replacement with Porcine Bioprosthetic Valves in Dogs
}

\author{
Kazuaki TAKASHIMA ${ }^{1) *}$, Aiko SODA ${ }^{2)}$, Ryou TANAKA ${ }^{2)}$ and Yoshihisa YAMANE ${ }^{1,2)}$ \\ 1) Animal Clinical Research Foundation, 214-10 Yatsuya, Kurayoshi, Tottori 682-0025 and ${ }^{2)}$ Department of Veterinary Surgery, Faculty \\ of Agriculture, Tokyo University of Agriculture and Technology, 3-5-8 Saiwai, Fuchu, Tokyo 183-8509,Japan
}

(Received 12 December 2006/Accepted 19 April 2007)

\begin{abstract}
Porcine bioprosthetic valves cross-linked with glutaraldehyde and polyepoxy compound were newly developed for mitral valve replacement (MVR) in dogs. Five beagle dogs were performed a left thoracotomy and underwent MVR using the porcine bioprosthetic valves during cardiopulmonary bypass (CPB). A vein catheter inserted into right atrium and a vent catheter inserted into the right ventricle to drain. The hemodynamic conditions of $\mathrm{CPB}$ were excellent during surgery. The left atrial pressure was measured before and after MVR; there was no significant difference and it was normal. Thrombosis and the prosthetic valve regurgitation were not observed one week after MVR. Pressure half time (PHT) prolonged significantly $(\mathrm{P}<0.05)$ from $31.40 \pm 4.0$ msec presurgery to $99.20 \pm 19.4$ msec at seven days after MVR, although it indicated the normal range as the bioprosthetic valve. The symptom of the prosthetic valve failure was not observed. This study indicated that the MVR using porcine bioprosthetic valves under CPB might have been effective in dogs as a short-term evaluation.

KEY WORDS: bioprosthetic valve, canine cardiopulmonary bypass, mitral regurgitation, mitral valve replacement.
\end{abstract}

Mitral regurgitation (MR) is the most common cause of congested heart disease, occurring secondary to myxomatous degenerative mitral valve in dogs. In myxomatous degeneration, the valves are thickened and the valve leaflets become redundant; and secondary dilation of the valve annulus contributes to mitral regurgitation. MR progresses insidiously with time and develops into congestive heart failure [13]. In a retrospective study, where 1421 dogs were diagnosed with cardiovascular diseases, MR comprised $83 \%$ of all acquired heart diseases, excluding dirofilariasis. MR was seen more frequently in small breeds and the peak occurrence was observed in 10 to 12 years of age [30].

Medical therapy of MR is angiotensin converting enzyme (ACE) inhibitors, diuretics, vasodilators and digoxin administration. However, progressive congestive heart failure and death are the inevitable consequences of severe MR despite any optimal medical treatment [7]. In human medicine, the definitive treatment of MR is surgical correction. Surgical treatment of MR is mitral valve replacement (MVR) or mitral valve repair (MVr). MVr has become the procedure of choice for surgical treatment of MR because it provides better postoperative clinical outcome than MVR in human medicine [1]. A few studies on successful MVr for dogs also have been reported [8, 12]. However, MVr is more difficult to accomplish than MVR. Moreover, MVR is recommended for non-ischemic severe MR and for non-reparable acute symptomatic MR, advanced symptomatic status, systolic dysfunction and/or ventricular dysfunction [1, 17].

MVR can be conducted using either bioprosthetic valves or mechanical valves. Mechanical valves have excellent

\footnotetext{
* Correspondence to: Takashima, K., Animal Clinical Research Foundation, 214-10 Yatsuya, Kurayoshi, Tottori 682-0025, Japan

e-mail: takashima@ncn-k.net
}

durability but have thrombogenicity and require a lifetime anticoagulation therapy. If thrombosis happens, the valves will not function normally. On the other hand, bioprosthetic valves have limited durability but have low thrombogenicity and do not require long-term anticoagulation therapy [1, 22]. In dogs, mechanical valve replacement is so difficult to obtain a long-term survival rate because of a prosthetic valve thrombosis $[21,24]$. Therefore, bioprosthetic valves are recommended for dogs, and porcine bioprosthetic valves cross-linked with glutaraldehyde and polyepoxy compounds were newly developed for MVR. Glutaraldehyde and Polyepoxy compounds (Denacol EX-313) have been used for bioprosthetic valves to reduce thrombosis and tissue antigenicity $[19,20]$. In mitral valve recipients, the rate of thromboembolism during the first 10 days was significantly higher than after 10 days [9]. The first several days from the perioperative period are very important for the evaluation of the MVR.

The aims of this study were to evaluate the short-term performance of the newly developed porcine bioprosthetic valves and techniques of cardiopulmonary bypass (CPB) and MVR in dogs.

\section{MATERIALS AND METHODS}

Bioprosthetic valves: Porcine aortic valves, with similar dimensions to that of canine mitral valves, were used. The porcine aortic valves were obtained aseptically from the slaughterhouse. Fat and myocardium were politely removed from the valves. After the trimming, the valves were packed with cottons to prevent the aortic annulus and the leaflet deformation, and were immersed in 50\% ethanol solution. Within two weeks, the valves were fixed as follows. 
1. $0.2 \%$ protamine sulfate (for $30 \mathrm{~min}$ at room temperature)

2. $0.5 \%$ glutaraldehyde (for $30 \mathrm{~min}$ at room temperature)

3. $4 \%$ polyepoxy compounds* (for $48 \mathrm{hr}$ at $40^{\circ} \mathrm{C}$ )

4. Washing with sterile water (for $24 \mathrm{hr}$ at room temperature)

5. $1 \%$ heparin sodium (for $1 \mathrm{hr}$ at $37-40^{\circ} \mathrm{C}$ )

* Denacol EX-313, Nagase Chemicals Ltd., Osaka Japan The valves were preserved in $70 \%$ ethanol solution.

The valves were trimmed again, the porcine aortic wall was removed and only the leaflets and commissures were left. The stainless stents appropriate for the annular and the commissure were made with Kirschner stainless wires of $0.6 \mathrm{~mm}$ in diameter. The annular and the commissure were sewn on the stainless stents. After covering and suturing the stents by Dacron fabrics, porcine bioprosthetic valves were completed (Fig. 1). The porcine bioprosthetic valves were then preserved in $30 \%$ ethanol solution under cold storage. At least two hours before transplantation, they were washed by sterilization using physiological saline solution to remove the alcohol.

Animals: Five male apparently healthy beagle dogs (1 year of age, 10-14 kg) were used. The dogs were subjected to clinical examinations, complete blood counts, heartworm antigen tests, blood chemistry, thoracic radiography, electrocardiography, phonocardiography and echocardiography. No abnormal findings were observed in the dogs.

In this study, all dogs were housed in individual runs. Standard environmental conditions of $22-23^{\circ} \mathrm{C}$ ambient temperature and $40-50 \%$ relative humidity were maintained. The dogs were given balanced maintenance diets and fresh water. During all phases of this study, the laboratory animals were handled and care for in accordance with the standards established by Tokyo University of Agriculture and Technology as described in its "Guide for the care and use of laboratory animals".

Anesthesia and CPB system: In this study, the basic protocols for anesthesia and CPB system used were those performed previously by our group [10, 23, 28].
Technique of $C P B$ and MVR: An arterial pressure catheter and an arterial catheter for $\mathrm{CPB}$ were each inserted into a different femoral artery. A central venous pressure catheter was inserted via the jugular vein. A left lateral thoracotomy was performed via the fifth intercostal space and the pericardium was exposed. After preparing a pericardial tent, a twostage single catheter (18-24FG) was inserted into the right atrium, and a $12 \mathrm{FG}$ vent catheter was inserted into the right ventricle to drain the blood from the heart. A cardioplegia cannula was positioned at the root of the aorta [25]. An arterial catheter for $\mathrm{CPB}$, a two-stage single catheter and a $12 \mathrm{FG}$ vent catheter were connected to CPB system.

After hemodynamics was stabilized in a partial perfusion, the ascending aorta and pulmonary artery were crossclamped and total perfusion was achieved. Cold Young's solution was administered from a cardioplegia cannula and cardiac arrest was achieved. GIK solution was also administered via a cardioplegia cannula every $20 \mathrm{~min}$ while the aorta was cross-clamped [25]. The dogs were cooled to approximately $26-28^{\circ} \mathrm{C}$, and ice slush saline was used to cool the heart regionally [23].

The mitral valve was approached via an incision in the left atrium. All mitral valves and the chordae tendineae were excised. Three 5-0 polypropylene sutures were preplaced through the mitral annulus from the atrial to the ventricular side of the annulus and then through the bioprosthetic ring from the ventricular to the atrial side. The bioprosthetic valve was seated into the mitral annulus with its left coronary cusp oriented to the left ventricular outflow tract. After the continuous suture of the bioprosthetic ring and mitral annulus, atriotomy was closed and air was removed from the left side of the heart (Fig. 2). After the aortic cross clamp was removed, the heartbeat resumed with cardiac massage and/or internal electrical defibrillation. As the atrial pressure rose, gradual cessation of $\mathrm{CPB}$ was initiated. After discontinuation of CPB, all catheters were removed. The left atrial pressure was measured directly before and after MVR, a thoracostomy tube was placed and thoracotomy incision was closed routinely.
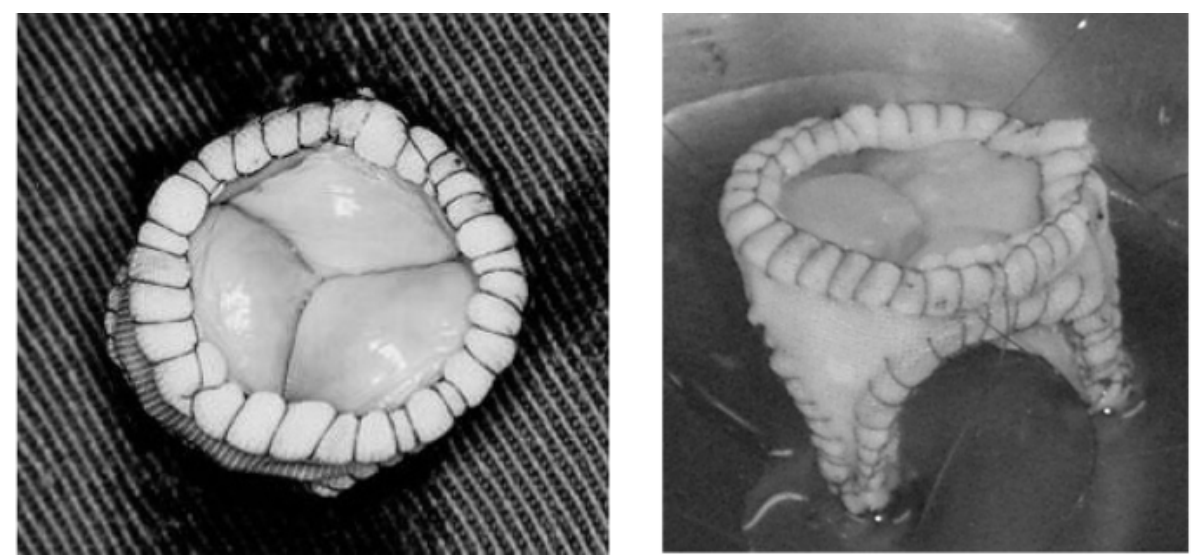

Fig. 1. The porcine bioprosthetic valve, cross-linked with glutaraldehyde and polyepoxy compound, used in this study. 


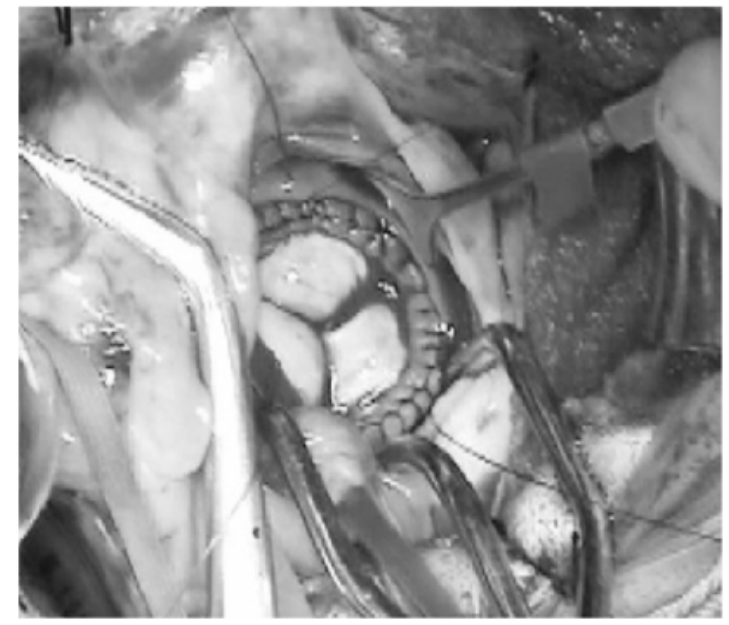

Fig. 2. Intraoperative view of mitral valve replacement using the porcine bioprosthetic valve under cardiopulmonary bypass.

Postoperative management: Ampicillin sodium $50 \mathrm{mg} / \mathrm{kg}$ IV (QID) and heparin sodium 50-100 U/kg SC (TID-QID) were administered postoperatively. Oral administration of dipyridamole and warfarin were initiated 2-5 days after operation. Warfarin was administered at $0.1 \mathrm{mg} / \mathrm{kg}$ (BID) and its dose was adjusted according to the results of the Thrombotest $(20 \%)$. Postoperative pain was managed by administration of butorphanol tartrate $(0.2-0.8 \mathrm{mg} / \mathrm{kg}$ intramuscularly) as necessary.

Clinical examination: The examinations included thoracic radiography, phonocardiography, electrocardiography, echocardiography and blood tests (CBC, TP, AST, ALT, ALP, BUN, Cre, TBil, Glu, TCho and CK) were conducted before and seven days after MVR. Vertebral heart size (VHS) and cardio thoracic ratio (CTR) were calculated by thoracic radiography $[7,13]$.

The annular diameter of the mitral valve before surgery was measured by two-dimensional echocardiography to choose the bioprosthetic valve. Diastolic atrioventricular pressure gradients (Bernoulli equation) were obtained from the continuous-wave Doppler signal of mitral flow velocity [3]. Pressure-half time (PHT), which is the time interval during which the atrioventricular pressure gradient fell to half its initial value, was calculated from these tracings. Mitral valve area (MVA) was calculated by the continuity equation (MVA=220/PHT) [3]. Left ventricular end-diastolic diameter (LVEdD), left ventricular end-systolic diameter (LVEsD), left ventricular posterior wall thickness in diastole (LVPWd), left ventricular posterior wall thickness in systole (LVPWs), interventricular septum thickness in diastole (IVSd), interventricular septum thickness in systole (IVSs) and fractional shortening (FS) were obtained by Mmode echocardiography [3, 7]. Movements of bioprosthetic valves were also observed immediately after MVR.

Differences in the examinations conducted were determined by paired- $t$ test, and statistical significance was established at $\mathrm{p}<0.05$.

\section{RESULTS}

Surgical evaluation: In a left lateral thoracotomy, the insertion of a two-stage single catheter into the right atrium and a vent catheter into the right ventricle were performed smoothly. The volume of blood flow to the CPB system from these catheters (venous drainage) was enough to perform CPB.

All dogs underwent MVR using the porcine bioprosthetic valves; the annular diameter of the measured mitral valve was $22.50 \pm 0.9$ (mean \pm SE) mm by echocardiography and the diameter of the porcine bioprosthetic valve used was $23.75 \pm 0.8 \mathrm{~mm}$. The mitral valve was approached easily via an incision in the left atrium. The porcine bioprosthetic valve was seated into the mitral annulus, and was sutured tightly. The handling of the porcine bioprosthetic valve was excellent, and was easy to suture. CPB time was $84.00 \pm 9.7$ min, and cardiac arrest time was $42.20 \pm 1.9 \mathrm{~min}$. Time from release of aortic cross clamp to cessation of CPB was $34.00 \pm 7.3 \mathrm{~min}$. The lowest rectal temperature was $27.32 \pm$ $0.3^{\circ} \mathrm{C}$, while the lowest esophagus temperature was $28.10 \pm$ $0.3^{\circ} \mathrm{C}$ (Table 1). These surgical times were almost similar because MVR and CPB were conducted smoothly in all dogs. The left atrial pressure, prior to MVR was $5.41 \pm 0.5$ $\mathrm{mmHg}$, post MVR was $8.64 \pm 2.3 \mathrm{mmHg}$; there was no significant difference observed. It was considered that the valve functioned normally.

Postsurgical evaluation: Blood examination, phonocardiography and electrocardiography were not significantly different before and seven days after surgery, the values were in the normal range. In the thoracic radiography, VHS and CTR were not significantly different before and seven days after surgery $(9.98 \pm 0.2$ and $10.04 \pm 0.2,62.96 \pm 0.7$

Table 1. Values of different parameters obtained during CPB* in dogs subjected to mitral valve replacement using porcine bioprosthetic valves

\begin{tabular}{ccccc}
\hline Case NO. & $\begin{array}{c}\text { CPB time } \\
(\mathrm{min})\end{array}$ & $\begin{array}{c}\text { Cardiac arrest time } \\
(\mathrm{min})\end{array}$ & $\begin{array}{c}\text { Lowest rectal } \\
\text { temperature }\left({ }^{\circ} \mathrm{C}\right)\end{array}$ & $\begin{array}{c}\text { Lowest esophagus } \\
\text { temperature }\left({ }^{\circ} \mathrm{C}\right)\end{array}$ \\
\hline 1 & 77 & 46 & 27.9 & 28.5 \\
2 & 91 & 46 & 27.2 & 28.6 \\
3 & 72 & 41 & 27.1 & 27.8 \\
4 & 62 & 36 & 27.9 & 28.5 \\
5 & 118 & 42 & 26.5 & 27.1 \\
\hline
\end{tabular}

* Cardiopulmonary bypass. 

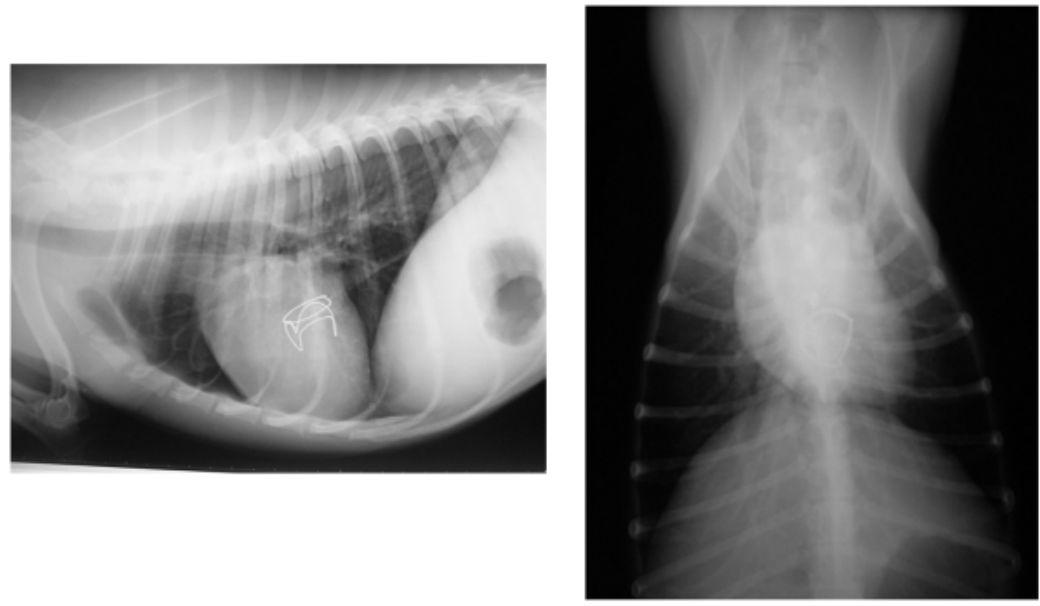

Fig. 3. Lateral (left) and dorsoventral (right) thoracic radiographs of a dog seven days after MVR.

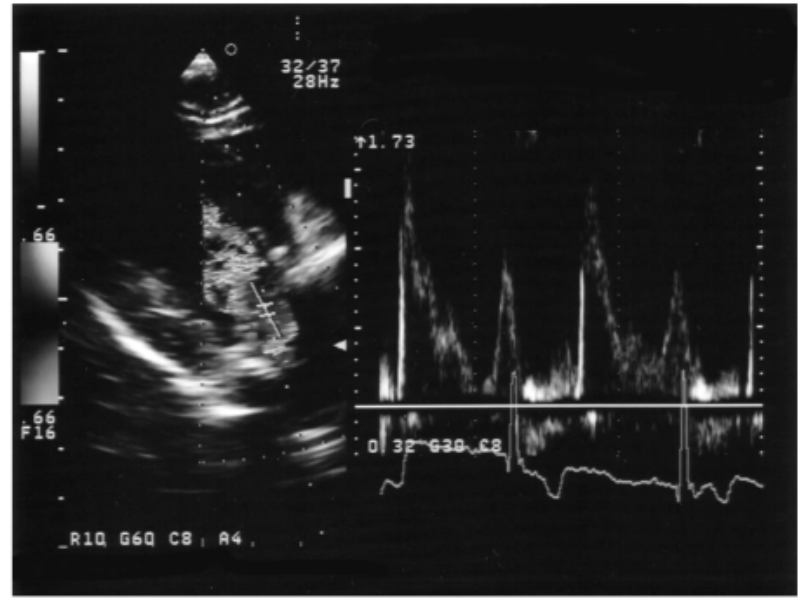

Fig. 4. Color and continuous-wave Doppler of the porcine bioprosthetic valve flow of a dog seven days after MVR.

and $63.70 \pm 1.8$, respectively ), the values were also in the normal range (Fig. 3).

In the echocardiography conducted immediately after MVR, the movements of the bioprosthetic valves were found to be smooth; laminar flow was also obtained seven days after surgery (Fig. 4). The prosthetic valve regurgitation and thrombosis were not observed immediately after MVR and seven days after MVR. Diastolic atrioventricular pressure gradient increased significantly $(\mathrm{P}<0.01)$ from 3.95 $\pm 0.8 \mathrm{mmHg}$ before surgery to $14.02 \pm 1.5 \mathrm{mmHg}$ seven days after surgery. PHT prolonged significantly $(\mathrm{P}<0.05)$ from $31.40 \pm 4.0 \mathrm{msec}$ before surgery to $99.20 \pm 19.4 \mathrm{msec}$ at seven days after surgery. MVA decreased significantly $(\mathrm{P}<0.05)$ from $7.44 \pm 0.8 \mathrm{~cm}^{2}$ before surgery to $2.68 \pm 0.6$ $\mathrm{cm}^{2}$ seven days after surgery. LVEdD, LVPWd, IVSd and IVSs were significantly $(\mathrm{P}<0.01)$ different before and seven days after surgery. No significant differences were
Table 2. M-Mode echocardiographic findings in dogs subjected to mitral valve replacement using porcine bioprosthetic valves

\begin{tabular}{lcc}
\hline & Before surgery $(\mathrm{mm})$ & Seven days after surgery $(\mathrm{mm})$ \\
\hline LVEdD & $33.68 \pm 1.1$ & $28.48 \pm 1.3^{*}$ \\
LVEsD & $20.92 \pm 0.7$ & $16.80 \pm 1.8$ \\
LVPWd & $6.56 \pm 0.2$ & $10.56 \pm 0.8^{*}$ \\
LVPWs & $10.52 \pm 0.7$ & $13.72 \pm 1.2$ \\
IVSd & $7.28 \pm 0.7$ & $10.36 \pm 0.4^{*}$ \\
IVSs & $10.44 \pm 0.4$ & $14.08 \pm 0.9^{*}$
\end{tabular}

Values are expressed as mean \pm standard error. LVEdD: left ventricular end-diastolic diameter, LVEsD: left ventricular endsystolic diameter, LVPWd:left ventricular posterior wall thickness in diastole, LVPWs: left ventricular posterior wall thickness in systole, IVSd: interventricular septum thickness in diastole, IVSs: interventricular septum thickness in systole. $* \mathrm{P}<0.01$ (vs. before surgery).

observed between before and after surgical LVEsD, LVPWs and $\mathrm{FS}$ values $(\mathrm{P}>0.05)($ Table 2$)$.

\section{DISCUSSION}

The cardiopulmonary bypass system has been used for over ten years in various canine congenital heart diseases in this group $[23,28,29]$. In a median sternotomy or a right lateral thoracotomy, venous catheters were generally inserted through the right atrium into a cranial vena cava and a caudal vena cava to drain the blood from the heart to CPB system [21, 23, 29]. But in this study, we performed MVR with a left lateral thoracotomy because the approach to the mitral valve via a left atrium incision was very easy. However, there was a big difficulty to perform CPB with a left thoracotomy, a cranial vena cava and a caudal vena cava were not seen from this side and venous catheters were not able to be inserted into the vena cava. Therefore, we tried to use a two-stage single catheter inserted into the right atrium and a vent catheter inserted into the right ventricle to drain. 
The volume of blood flow to the CPB system from these catheters was enough to perform CPB and the hemodynamics condition was excellent. The annular diameter of the mitral valve was measured by two-dimensional echocardiography before surgery to choose the bioprosthetic valve. The value was almost accurate, the porcine bioprosthetic valve was able to be inserted without trouble. The bioprosthetic sewing ring covered with Dacron fabrics, was easy to suture the mitral annular. The handling of the porcine bioprosthetic valve was excellent. CPB time, cardiac arrest time and time from release of aortic cross clamp to cessation of CPB were not very different respectively, because CPB and MVR were performed smoothly and stabilized.

There are different types of bioprosthetic valves for humans, including Hancock II bioprostheses (porcine bioprosthesis), Carpentier-Edwards bovine pericardial valves and Mosaic bioprosthesis (porcine bioprosthesis); the longterm prognoses of these valves have been reported only in human medicine $[4,6]$. Glutaraldehyde has been widely used as a tissue fixative for these bioprosthetic valves and vascular grafts due to its ability to produce collagen crosslinking, reduce tissue antigenicity and sterilize the valves [27]. Bioprosthetic valves have a possibility of the prosthetic valve failure due to calcification, pannus formation and tissue degeneration; several reagents have been extensively investigated to inhibit them $[5,22]$. Polyepoxy compound (Denacol EX-313) was developed in order to reduce calcification, degeneration and thrombosis $[20,26]$. Previous studies by this group have demonstrated the excellent durability of polyepoxy compound fixed bovine jugular vein graft for reconstruction of right ventricular outflow tract in dogs [16]. An apico-aortic valve conduit using canine aortic bioprosthetic valve was also developed for surgical treatment of aortic stenosis in dogs. The canine aortic bioprosthetic valve was fixed with glutaraldehyde and epoxy compound and was found to be effective for aortic stenosis [11]. In this study, the bioprosthetic valve was newly developed with the porcine aortic valve, because the size of valve is similar to canine mitral valves, the stable supply is possible and porcine bioprosthesis are used in human medicine. The rate of thromboembolism in patients undergoing bioprosthetic replacement of the mitral valve, during the first 10 days was $55 \%$ /year, 11 to 90 days was $10 \%$ year and after 90 days was $2.4 \%$ /year. The complication of MVR was significantly higher than after 10 days [9]. The first several days from surgery are very important for the evaluation of MVR. Therefore, as the first step, we evaluated performance of the newly developed porcine bioprosthetic valves one week after the MVR.

In this study, there was no significant increase in the left atrial pressure before and after the MVR, the movement of the bioprosthetic valve was smooth and the laminar flow was obtained seven days after MVR. Complications, such as acute regurgitation of the prosthetic valve and the thrombogenesis, were not observed in all dogs. After MVR, diastolic atrioventricular pressure gradient and PHT increased significantly to $14.02 \pm 1.5 \mathrm{mmHg}$ and $99.20 \pm 19.4 \mathrm{msec}$, respectively. The values deviated from the normal canine values, and were similar to those observed in natural occurred canine mitral stenosis (MS) $[3,15]$. On the other hand, there is a criterion of the bioprosthetic valve, and the bioprosthetic valve is evaluated based on this $[2,31]$. The diastolic atrioventricular pressure gradient was greater than $16 \mathrm{mmHg}$ and PHT greater than $180 \mathrm{msec}$, which suggests abnormal functioning of the bioprosthetic valve $[2,18]$. Thus, the values of the atrioventricular pressure gradient and PHT after MVR in this study were suggested to be normally functional as the bioprosthetic valve. Mitral valve area can be calculated using PHT. The value was also in the normal range as the bioprosthetic valve, although a significant reduction was observed compared to the value before MVR [31].

A significant change in the size of the left ventricle such as decreases of LVEdD was observed after MVR. In 14 dogs of MS, there was no relationship observed between left ventricular size and MS, because the left ventricular size was normal $(6 / 14)$, increased $(5 / 14)$, or decreased $(3 / 14)$, as assessed by measurement of LVEdD [15]. In humans, LVEdD was usually reduced after MVR and MVr, because left ventricular volume overload was eliminated [14]. LVEdD increases with progression of congestive heart failure, so the decrease of LVEdD after the MVR and MVr usually shows the effectiveness of the operation. In this study, it was difficult to evaluate whether the change in size of the left ventricle after MVR was influenced by MVR or by the porcine bioprosthetic valve, because apparently healthy dogs were used. However, left ventricular systolic function was kept normal after MVR, because FS was in the normal range after MVR.

We concluded that MVR procedures by a left thoracotomy under $\mathrm{CPB}$ and the use of the newly developed porcine bioprosthetic valves might have been effective in dogs as a short-term evaluation. The long-term evaluation of antithrombotic, durability and the anti-acquired immune characteristics of the bioprosthetic valve need to be investigated.

ACKNOWLEDGEMENT. We thank the members of the Department of Veterinary Surgery, Faculty of Agriculture, Tokyo University of Agriculture and Technology, and Animal Clinical Research foundation for their support in this study.

\section{REFERENCES}

1. Arai, T. 2005. Heart Surgery. Tokyo, Igakushoin (in Japanese).

2. Bernardes, L., Abreu, J., Soares, R., Matos, P., Ramos, J. S. and Salomao, S. 1990. Mitral prostheses with and without dysfunction: evaluation using 2-D Doppler echocardiography. Rev. Port. Cardiol. 9: 973-979.

3. Boon, J. A. 1998. Congenital heart disease, pp. 383-445. In: Manual of Veterinary Echocardiography (Boon, J. A.ed.). Baltimore, Maryland: Lippincott Williams \& Wilkins.

4. Borger, M. A., Ivanov, J., Armstrong, S., Christie-Hrybinsky, D., Feindel, C. M. and David, T. E. 2006. Twenty-year results of the Hancock II bioprosthesis. J. Heart Valve Dis. 15: 49-55; 
discussion 55-46.

5. Bottio, T., Thiene, G., Pettenazzo, E., Ius, P., Bortolotti, U., Rizzoli, G., Valfre, C., Casarotto, D. and Valente, M. 2003. Hancock II bioprosthesis: a glance at the microscope in midlong-term explants. J. Thorac. Cardiovasc. Surg. 126: 99-105.

6. Eichinger, W. B., Botzenhardt, F., Gunzinger, R., Kemkes, B. M., Sosnowski, A., Maiza, D., Coto, E. O. and Bleese, N. 2002. European experience with the Mosaic bioprosthesis. J. Thorac. Cardiovasc. Surg. 124: 333-339.

7. Fox, P. R., Sisson, D. and Moise, N. S. 1999. pp. 536-555. Textbook of Canine and Feline Cardiology. Philadelphia: W.B.Saunders.

8. Griffiths, L. G., Orton, E. C. and Boon, J. A. 2004. Evaluation of techniques and outcomes of mitral valve repair in dogs. $J$. Am. Vet. Med. Assoc. 224: 1941-1945.

9. Heras, M., Chesebro, J. H., Fuster, V., Penny, W. J., Grill, D. E., Bailey, K. R., Danielson, G. K., Orszulak, T. A., Pluth, J. R., Puga, F. J. et al. 1995. High risk of thromboemboli early after bioprosthetic cardiac valve replacement. J. Am. Coll. Cardiol. 25: 1111-1119.

10. Hirao, H., Hoshi, K., Kobayashi, M., Shimizu, M., Shimamura, S., Tanaka, R., Machida, N., Maruo, K. and Yamane, Y. 2004. Surgical correction of subvalvular aortic stenosis using cardiopulmonary bypass in a dog. J. Vet. Med. Sci. 66: 559-562.

11. Hirao, H., Inoue, T., Hoshi, K., Kobayashi, M., Shimamura, S., Shimizu, M., Tanaka, R., Takashima, K., Mori, Y., Noishiki, Y. and Yamane, Y. 2005. An experimental study of apico-aortic valved conduit (AAVC) for surgical treatment of aortic stenosis in dogs. J. Vet. Med. Sci. 67: 357-362.

12. Kerstetter, K. K., Sackman, J. E., Buchanan, J. W., Bright, J. M., Krahwinkel, D. J., Bright, R. M., Lorinson, D. and Gompf, R. E. 1998. Short-term hemodynamic evaluation of circumferential mitral annuloplasty for correction of mitral valve regurgitation in dogs. Vet. Surg. 27: 216-223.

13. Kittleson, M. D. and Kienle, R. D. 1998. pp. 297-318. Small Animal Cardiovascular Medicine. St. Louis: Mosby.

14. Kouris, N., Ikonomidis, I., Kontogianni, D., Smith, P. and Nihoyannopoulos, P. 2005. Mitral valve repair versus replacement for isolated non-ischemic mitral regurgitation in patients with preoperative left ventricular dysfunction. A long-term follow-up echocardiography study. Eur. J. Echocardiogr. 6: 435442.

15. Lehmkuhl, L. B., Ware, W. A. and Bonagura, J. D. 1994. Mitral stenosis in 15 dogs. J. Vet. Intern. Med. 8: 2-17.

16. Matsumoto, H., Sugiyama, S., Shibazaki, A., Tanaka, R., Takashima, K., Noishiki, Y. and Yamane, Y. 2003. A long term comparison between Denacol EX-313-treated bovine jugular vein graft and ultrafine polyester fiber graft for reconstruction of tight ventricular outflow tract in dogs. J. Vet. Med. Sci. 65: $363-368$.

17. Moffatt-Bruce, S. D. and Jamieson, W. R. 2004. Long-term performance of prostheses in mitral valve replacement. J. Cardiovasc. Surg. (Torino) 45: 427-447.

18. Nakamura, K., Matsumura, K., Satomi, G., Sakai, K., Ishizuka,
N., Mori, K., Shiina, T., Kikuchi, N., Hirosawa, K. and Takao, A. 1986. Doppler evaluation of porcine mitral valve dysfunction. J. Cardiogr. 16: 929-939.

19. Noishiki, Y., Miyata, T. and Kodaira, K. 1986. Development of a small caliber vascular graft by a new crosslinking method incorporating slow heparin release collagen and natural tissue compliance. ASAIO Trans. 32: 114-119.

20. Okoshi, T., Noishiki, Y., Tomizawa, Y., Morishima, M., Taira, T., Kawai, T., Itoh, H., Miyata, T. and Koyanagi, H. 1990. A new bioprosthetic cardiac valve with reduced calcification. ASAIO Trans. 36: M411-414.

21. Orton, E. C., Hackett, T. B., Mama, K. and Boon, J. A. 2005. Technique and outcome of mitral valve replacement in dogs. $J$. Am. Vet. Med. Assoc. 226: 1508-1511, 1500.

22. Rizzoli, G., Bottio, T., Thiene, G., Toscano, G. and Casarotto, D. 2003. Long-term durability of the Hancock II porcine bioprosthesis. J. Thorac. Cardiovasc. Surg. 126: 66-74.

23. Shimamura, S., Kutsuna, H., Shimizu, M., Kobayashi, M., Hirao, H., Tanaka, R., Takashima, K., Machida, N. and Yamane, Y. 2006. Comparison of right atrium incision and right ventricular outflow incision for surgical repair of membranous ventricular septal defect using cardiopulmonary bypass in dogs. Vet. Surg. 35: 382-387.

24. Starr, A., Fessler, C. L., Grunkemeier, G. and He, G. W. 2002. Heart valve replacement surgery: past, present and future. Clin. Exp. Pharmacol. Physiol. 29: 735-738.

25. Takeuchi, E., Murase, M., Tanaka, M., Hibi, M., Yasuura, K., Ogawa, K., Hotta, A., Saigusa, H., Yoshida, K. and Abe, T. 1986. Replenishment of Young-GIK cardioplegia under the reaortic cross clamp. Nippon Kyobu Geka Gakkai Zasshi 34: 447-452 (in Japanese with English summary).

26. Tomizawa, Y., Moon, M. R., DeAnda, A., Castro, L. J., Kosek, J. and Miller, D. C. 1994. Coronary bypass grafting with biological grafts in a canine model. Circulation 90: 160-166.

27. Vongpatanasin, W., Hillis, L. D. and Lange, R. A. 1996. Prosthetic heart valves. New Engl. J. Med. 335: 407-416.

28. Yamagata, S., Yamane, Y., Shibazaki, A., Matsumoto, H., Takashima, K., Takashima, S., Kuno, Y., Masada, S., Masuda, Y., Oguchi, Y. and Noishiki, Y. 1998. Studies on the development and clinical use of cardiopulmonary bypass system for small Animals. J. Anim. Clin. Med. 6: 13-25 (in Japanese with English summary).

29. Yamane, Y., Shibasaki, F., Kouzuki, S., Tsuruno, M., Matsumoto, H., Taguchi, J., Kouno, S., Koie, H., Kishigami, Y., Aoyama, S. and Noishiki, Y. 1993. A case of surgical correction for pulmonic stenosis by using a patch graft under the extracorporeal circulation in dog. J. Anim. Res. Found 1: 33-40 (in Japanese with English summary).

30. Yasutake, S., Takashima, K. and Yamane, Y. 2005. A retrospective study of canine and feline cardiovascular disease in 1521 cases. J. Anim. Clin. Med. 14: 123-132 (in Japanese with English summary).

31. Yoshikawa, J. 2005. Clinical Echocardiography. Tokyo: Buneido (in Japanese ). 